\title{
Servicios otorgados en unidades de la Secretaría de Salud, 1999
}

L

a Secretaría de Salud (SSA)

cuenta con el Sistema de Información en Salud para Población Abierta (SISPA), constituido por cuatro subsistemas, los que dan respuesta a las necesidades de información en salud. El SISPA, mediante dichos subsistemas, tiene como propósito generar e integrar información referente a: a) recursos disponibles en la SSA, b) población, c) daños a la salud y d) prestación de servicios.

Este último subsistema capta información referente a los servicios otorgados a la población demandante, datos que son obtenidos mensualmente por medio del informe que rinde cada unidad aplicativa de la SSA en las 32 entidades federativas y que es enviado en forma de concentrado por cada entidad a la
Dirección General de Estadística e Informática.

La información permite conocer la cobertura e impacto del servicio, así como la evaluación de la operación; además, proporciona apoyo a tomadores de decisiones e investigadores.

Se presenta a continuación información anual captada por el SISPA referente a 1999. Una parte de la información muestra las atenciones brindadas, que está constituida por la consulta externa, hospitalización y los servicios auxiliares de diagnóstico, y la otra, contiene tabulados con las distintas poblaciones según los servicios de los que han hecho uso, como aquellas que se refieren a los menores de cinco años, la atención materna, los servicios de pla- nificación familiar, los accidentes y las detecciones de los diferentes padecimientos.

Debido a la gran cantidad de información que capta este subsistema, la información aquí presentada se refiere a una pequeña muestra de lo que ofrece el SISPA; dicha información se encuentra disponible en la página Web de la SSA y en las publicaciones de la Dirección General de Estadística e Informática (sita en Leibnitz 20, 3er. piso, colonia Nueva Anzures, 11590 México, D.F.), como son: el Anuario Estadístico de la propia Secretaría de Salud y el Boletín Mensual del SISPA, además en las tabulaciones que se generan mensualmente por cada programa sustantivo de la SSA. 


\section{Consulta externa otorgada. México, 1999}

\begin{tabular}{|c|c|c|c|c|c|c|c|c|}
\hline \multirow[b]{2}{*}{ Entidad federativa } & \multirow[b]{2}{*}{ Total } & \multicolumn{3}{|c|}{ Enfermedades } & \multirow[b]{2}{*}{$\begin{array}{l}\text { Consulta } \\
\text { a sanos }\end{array}$} & \multirow[b]{2}{*}{$\begin{array}{l}\text { Planificación } \\
\text { familiar* }\end{array}$} & \multirow[b]{2}{*}{$\begin{array}{l}\text { Salud } \\
\text { bucal }\end{array}$} & \multirow[b]{2}{*}{$\begin{array}{l}\text { Salud } \\
\text { mental }\end{array}$} \\
\hline & & $\begin{array}{l}\text { Transmi- } \\
\text { sibles }\end{array}$ & $\begin{array}{c}\text { Crónico } \\
\text { degenerativas }\end{array}$ & Otras & & & & \\
\hline Estados Unidos Mexicanos & 65987573 & 13986689 & 3128784 & 21243737 & 16901528 & 5474397 & 4668678 & 583760 \\
\hline Aguascalientes & 547734 & 128998 & 41967 & 155913 & 117505 & 38993 & 49683 & 14675 \\
\hline Baja California & 570014 & 112335 & 45572 & 184593 & 107723 & 63858 & 49342 & 6591 \\
\hline Baja California Sur & 320207 & 62954 & 26175 & 91044 & 82180 & 33763 & 19033 & 5058 \\
\hline Campeche & 656281 & 172501 & 25782 & 207586 & 155449 & 52589 & 42374 & 0 \\
\hline Coahuila & 1062513 & 276437 & 70308 & 318439 & 176518 & 113343 & 100162 & 7306 \\
\hline Colima & 451455 & 113203 & 30781 & 150222 & 84637 & 36303 & 33826 & 2483 \\
\hline Chiapas & 2217484 & 552409 & 65473 & 737277 & 441859 & 197541 & 211775 & 11150 \\
\hline Chihuahua & 1205956 & 224104 & 86380 & 423155 & 294050 & 109113 & 59604 & 9550 \\
\hline Distrito Federal & 6478922 & 677286 & 167736 & 3478730 & 921258 & 222116 & 808947 & 202849 \\
\hline Durango & 793826 & 200756 & 58558 & 239662 & 134669 & 98232 & 51860 & 10089 \\
\hline Guanajuato & 2608623 & 727248 & 108249 & 725381 & 716620 & 187687 & 130075 & 13363 \\
\hline Guerrero & 3847265 & 877384 & 125119 & 1052849 & 1281081 & 390134 & 113357 & 7341 \\
\hline Hidalgo & 2207337 & 575146 & 93452 & 576440 & 593455 & 135614 & 226174 & 7056 \\
\hline Jalisco & 3445427 & 666481 & 258179 & 1017942 & 998738 & 278155 & 183769 & 42163 \\
\hline México & 8916086 & 1842524 & 245051 & 1680306 & 3161196 & 1482353 & 486682 & 17974 \\
\hline Michoacán & 2821098 & 741456 & 185395 & 945550 & 545555 & 164814 & 226133 & 12195 \\
\hline Morelos & 1213340 & 353462 & 48396 & 413720 & 216814 & 81403 & 95478 & 4067 \\
\hline$N$ ayarit & 910064 & 174734 & 67300 & 235035 & 296604 & 89266 & 43250 & 3875 \\
\hline N uevo León & 2273583 & 455299 & 174257 & 645155 & 637328 & 155125 & 176589 & 29830 \\
\hline 0 axaca & 2400307 & 640026 & 96218 & 770167 & 557512 & 164904 & 158666 & 12814 \\
\hline Puebla & 2580426 & 569630 & 121180 & 625935 & 805305 & 175500 & 272020 & 10856 \\
\hline Q uerétaro & 1007660 & 254544 & 36075 & 299655 & 299827 & 45320 & 62129 & 10110 \\
\hline Q uintana Roo & 629543 & 160561 & 24781 & 182744 & 165402 & 65705 & 30350 & 0 \\
\hline San Luis Potosí & 1293256 & 242512 & 106368 & 324627 & 391067 & 89442 & 133404 & 5836 \\
\hline Sinaloa & 1305005 & 281492 & 85033 & 409299 & 341415 & 86405 & 83261 & 18100 \\
\hline Sonora & 1330529 & 310262 & 80606 & 458671 & 269072 & 92865 & 85188 & 33865 \\
\hline Tabasco & 2836310 & 559962 & 101597 & 886814 & 852404 & 169232 & 246698 & 19603 \\
\hline Tamaulipas & 1631125 & 353812 & 145985 & 600158 & 347935 & 99440 & 70851 & 12944 \\
\hline Tlaxcala & 1067915 & 255281 & 44062 & 275914 & 343925 & 82981 & 61311 & 4441 \\
\hline Veracruz & 4017597 & 878815 & 230825 & 1382298 & 1001488 & 289343 & 218514 & 16314 \\
\hline Yucatán & 1181766 & 313800 & 58148 & 354752 & 303468 & 77304 & 46629 & 27665 \\
\hline Zacatecas & 1040683 & 231275 & 73776 & 310793 & 259469 & 100539 & 61234 & 3597 \\
\hline Institutos nacionales & 1118236 & 0 & 0 & 1082911 & 0 & 5015 & 30310 & 0 \\
\hline
\end{tabular}

* Se refiere a consultas cuyo único motivo fue planificación familiar. Difiere del presentado en el cuadro VI que contiene atenciones colaterales y lo correspondiente a Estrategia de Extensión de Cobertura

Fuente: Sistema de Información en Salud para PoblaciónA bierta (SISPA), D irección General de Estadística e Informática (D GEI), Subsecretaría de Prevención y Control de Enfermedades, Secretaría de Salud (SSA), México 


\section{Cuadro II \\ Servicios de hospitalización.* México, 1999}

\begin{tabular}{|c|c|c|c|c|c|c|c|c|}
\hline \multirow[b]{2}{*}{ Entidad federativa } & \multirow[b]{2}{*}{ Egresos } & \multirow[b]{2}{*}{ Días paciente } & \multirow[b]{2}{*}{ Días estancia } & \multirow[b]{2}{*}{ Intervenciones quirúrgicas } & \multirow[b]{2}{*}{ Porcentaje de ocupacion } & \multirow[b]{2}{*}{ Promedio de días estancia } & \multicolumn{2}{|c|}{ Mortalidad hospitalaria } \\
\hline & & & & & & & Total & Ajustada \\
\hline Estados Unidos Mexicanos & 1402808 & 6581636 & 5883225 & 806710 & 66.53 & 4.19 & 26.31 & 14.17 \\
\hline Aguascalientes & 27636 & 112963 & 91603 & 11577 & 77.29 & 3.31 & 14.87 & 9.81 \\
\hline Baja California & 25582 & 113523 & 91230 & 13991 & 98.54 & 3.57 & 45.89 & 27.01 \\
\hline Baja California Sur & 8989 & 39218 & 37779 & 6212 & 57.04 & 4.20 & 29.15 & 16.91 \\
\hline Campeche & 14471 & 41881 & 43141 & 8285 & 59.05 & 2.98 & 19.76 & 10.92 \\
\hline Coahuila & 19564 & 73665 & 64810 & 8968 & 52.33 & 3.31 & 16.87 & 8.79 \\
\hline Colima & 13634 & 40646 & 39997 & 7628 & 56.74 & 2.93 & 21.71 & 11.15 \\
\hline Chiapas & 49117 & 183864 & 145163 & 30716 & 88.67 & 2.96 & 31.46 & 15.19 \\
\hline Chihuahua & 43041 & 179434 & 164343 & 19595 & 70.00 & 3.82 & 32.18 & 11.04 \\
\hline Distrito Federal & 197035 & 1101890 & 1055937 & 125954 & 59.50 & 5.36 & 22.38 & 13.15 \\
\hline Durango & 24427 & 115224 & 95382 & 12537 & 70.50 & 3.90 & 28.57 & 16.21 \\
\hline Guanajuato & 59461 & 212703 & 179527 & 45613 & 71.88 & 3.02 & 20.80 & 12.11 \\
\hline Guerrero & 40838 & 155185 & 128597 & 24790 & 66.11 & 3.15 & 32.13 & 17.43 \\
\hline Hidalgo & 26695 & 197105 & 178683 & 14027 & 80.75 & 6.69 & 28.17 & 14.72 \\
\hline Jalisco & 61643 & 179456 & 177168 & 31632 & 49.80 & 2.87 & 12.99 & 8.03 \\
\hline México & 124308 & 808072 & 666164 & 80942 & 55.33 & 5.36 & 27.88 & 18.68 \\
\hline Michoacán & 53551 & 219143 & 192411 & 27899 & 73.43 & 3.59 & 27.62 & 16.90 \\
\hline Morelos & 28270 & 114569 & 91410 & 16340 & 93.60 & 3.23 & 29.61 & 15.60 \\
\hline $\mathrm{N}$ ayarit & 13853 & 44133 & 39882 & 8532 & 59.51 & 2.88 & 38.62 & 25.70 \\
\hline Nuevo León & 37939 & 160452 & 137211 & 21560 & 72.47 & 3.62 & 19.40 & 11.39 \\
\hline 0 axaca & 33443 & 151549 & 146638 & 18021 & 58.47 & 4.38 & 20.18 & 11.03 \\
\hline Puebla & 30185 & 223658 & 178720 & 17038 & 58.83 & 5.92 & 29.82 & 18.32 \\
\hline Q uerétaro & 24273 & 92639 & 72392 & 16010 & 74.81 & 2.98 & 29.42 & 17.72 \\
\hline Q uintana Roo & 13456 & 44874 & 39178 & 7467 & 64.92 & 2.91 & 22.00 & 13.75 \\
\hline San Luis Potosí & 24110 & 68125 & 68423 & 10420 & 82.64 & 2.84 & 19.91 & 9.12 \\
\hline Sinaloa & 31061 & 126167 & 104506 & 20621 & 83.44 & 3.36 & 30.39 & 20.57 \\
\hline Sonora & 52449 & 237653 & 199905 & 19670 & 68.69 & 3.81 & 18.23 & 11.19 \\
\hline Tabasco & 50765 & 236414 & 187447 & 34516 & 84.41 & 3.69 & 26.40 & 14.79 \\
\hline Tamaulipas & 62513 & 237948 & 212054 & 31078 & 60.53 & 3.39 & 30.20 & 17.72 \\
\hline Tlaxcala & 20529 & 52599 & 52789 & 9779 & 67.33 & 2.57 & 21.09 & 11.35 \\
\hline Veracruz & 91086 & 374288 & 373470 & 50903 & 68.49 & 4.10 & 31.66 & 16.31 \\
\hline Yucatán & 19688 & 133904 & 129062 & 11925 & 76.22 & 6.56 & 26.51 & 20.06 \\
\hline Zacatecas & 19354 & 67107 & 48955 & 9459 & 99.68 & 2.53 & 21.49 & 11.21 \\
\hline Institutos nacionales & 59842 & 441585 & 449248 & 33005 & 82.38 & 7.51 & 33.82 & ND \\
\hline
\end{tabular}

* Se refiere exclusivamente a unidades hospitalarias

ND: no disponible

Fuente: Sistema de Información en Salud para PoblaciónA bierta (SISPA), D irección G eneral de Estadística e Informática (D G El), Subsecretaría de Prevención y Control de Enfermedades, Secretaría de Salud (SSA), México 


\section{Cuadro III}

Servicios auXiLIares de diagnóstico. México, 1999

\begin{tabular}{|c|c|c|c|c|c|c|c|c|c|c|}
\hline \multirow[b]{2}{*}{ Entidad federativa } & \multicolumn{2}{|c|}{ Análisis clínicos } & \multicolumn{2}{|c|}{ Rayos X } & \multicolumn{2}{|c|}{ Anatomía patológica } & \multicolumn{2}{|c|}{ Electrocardiograma } & \multicolumn{2}{|c|}{ Ultrasonido } \\
\hline & Estudios & Personas & Estudios & Personas & Estudios & Personas & Estudios & Personas & Estudios & Personas \\
\hline Estados Unidos Mexicanos & 38148201 & 7974159 & 3071391 & 2332619 & 658382 & 460005 & 183849 & 175896 & 358445 & 348882 \\
\hline Aguascalientes & 687510 & 139246 & 96990 & 83254 & 3483 & 3438 & 5200 & 4914 & 4848 & 4846 \\
\hline Baja California & 563754 & 112060 & 64555 & 45882 & 9092 & 7222 & 12096 & 9834 & 4086 & 4065 \\
\hline Baja California Sur & 253889 & 81870 & 26464 & 22232 & 10001 & 10001 & 2375 & 2338 & 5069 & 5069 \\
\hline Campeche & 322040 & 89122 & 21459 & 17803 & 1339 & 1339 & 633 & 632 & 6333 & 6327 \\
\hline Coahuila & 615615 & 157357 & 35367 & 29115 & 5608 & 5608 & 1904 & 1828 & 9904 & 9900 \\
\hline Colima & 181048 & 80102 & 22009 & 19035 & 2294 & 2294 & 2386 & 2294 & 3696 & 3693 \\
\hline Chiapas & 999395 & 221127 & 64323 & 45540 & 17628 & 2483 & 2353 & 2070 & 4739 & 4701 \\
\hline Chihuahua & 1004114 & 224318 & 97386 & 78716 & 6891 & 6629 & 7273 & 6790 & 2831 & 2823 \\
\hline Distrito Federal & 7943876 & 1496930 & 662952 & 559798 & 217223 & 93009 & 36702 & 35806 & 93563 & 89221 \\
\hline Durango & 452002 & 132042 & 55596 & 47363 & 3196 & 3195 & 4095 & 3995 & 9863 & 9560 \\
\hline Guanajuato & 930612 & 257475 & 103635 & 85045 & 46071 & 45981 & 2367 & 2355 & 11352 & 11348 \\
\hline Guerrero & 1184306 & 196006 & 73776 & 48554 & 3443 & 3443 & 3583 & 3491 & 8901 & 8859 \\
\hline Hidalgo & 568177 & 170402 & 45686 & 37147 & 3471 & 3461 & 729 & 729 & 10773 & 10274 \\
\hline Jalisco & 1099942 & 297484 & 97309 & 69503 & 37804 & 37174 & 6513 & 6408 & 8085 & 7829 \\
\hline México & 2617833 & 649917 & 196436 & 149974 & 40319 & 40151 & 13594 & 13339 & 23046 & 22548 \\
\hline Michoacán & 925831 & 291621 & 108652 & 81127 & 8555 & 8432 & 5908 & 5908 & 17217 & 16935 \\
\hline Morelos & 606202 & 145176 & 59365 & 46860 & 5263 & 5262 & 5515 & 5515 & 3677 & 3677 \\
\hline $\mathrm{N}$ ayarit & 324325 & 101723 & 35798 & 24389 & 4696 & 1483 & 2455 & 2355 & 2040 & 2040 \\
\hline Nuevo León & 989782 & 239815 & 109360 & 88759 & 6772 & 6772 & 8112 & 8025 & 3003 & 2999 \\
\hline 0 axaca & 528519 & 145310 & 71085 & 46492 & 5948 & 3285 & 4723 & 4720 & 6919 & 6896 \\
\hline Puebla & 880585 & 246053 & 58627 & 44086 & 18610 & 10091 & 4299 & 3253 & 8905 & 8738 \\
\hline Q uerétaro & 518249 & 94263 & 52091 & 46381 & 4614 & 4200 & 2228 & 2228 & 8253 & 8241 \\
\hline Q uintana Roo & 384216 & 166321 & 24106 & 21227 & 1260 & 1260 & 655 & 655 & 5976 & 5976 \\
\hline San Luis Potosí & 591691 & 124461 & 29695 & 25967 & 1578 & 1578 & 3222 & 3186 & 3287 & 3277 \\
\hline Sinaloa & 736421 & 174983 & 70540 & 55728 & 22301 & 22190 & 3645 & 3645 & 13136 & 13090 \\
\hline Sonora & 1176981 & 265393 & 94438 & 69608 & 6927 & 6927 & 5946 & 4457 & 3492 & 3492 \\
\hline Tabasco & 1306692 & 249749 & 79776 & 62139 & 10112 & 10112 & 5121 & 5121 & 16612 & 16486 \\
\hline Tamaulipas & 1190797 & 334818 & 111893 & 88688 & 41026 & 40605 & 11719 & 11531 & 9907 & 9638 \\
\hline Tlaxcala & 440679 & 126903 & 34050 & 22602 & 5542 & 3040 & 1208 & 1208 & 7510 & 6919 \\
\hline Veracruz & 2241006 & 455709 & 122231 & 89110 & 30004 & 29059 & 11412 & 11404 & 25388 & 23410 \\
\hline Yucatán & 562125 & 147775 & 38417 & 32918 & 14289 & 14289 & 3188 & 3188 & 9104 & 9098 \\
\hline Zacatecas & 466832 & 107911 & 40427 & 34428 & 4822 & 4605 & 2690 & 2674 & 6930 & 6907 \\
\hline Institutos nacionales & 4853155 & 250717 & 266897 & 113149 & 58200 & 21387 & 0 & 0 & 0 & 0 \\
\hline
\end{tabular}

Fuente: Sistema de Información en Salud para Población A bierta (SISPA), D irección G eneral de Estadística e Informática (D GEI), Subsecretaría de Prevención y Control de Enfermedades, Secretaría de Salud (SSA), México 


\section{A tención a menores de cinco años. México, 1999}

\begin{tabular}{|c|c|c|c|c|c|c|c|c|}
\hline \multirow[t]{2}{*}{ Entidad federativa } & \multirow{2}{*}{$\begin{array}{l}\text { Consulta a } \\
\text { niño sano }\end{array}$} & \multicolumn{3}{|c|}{$\begin{array}{l}\text { Consulta según } \\
\text { presencia de desnutrición }\end{array}$} & \multicolumn{2}{|c|}{$\begin{array}{c}\text { Consultas enfermedad } \\
\text { diarreica aguda }{ }^{\ddagger} \\
\end{array}$} & \multicolumn{2}{|c|}{$\begin{array}{l}\text { Consultas infección } \\
\text { respiratoria aguda }{ }^{\ddagger}\end{array}$} \\
\hline & & Total & Sin desnutrición* & Con desnutrición & Total & Primera vez & Total & Primera vez \\
\hline Estados Unidos Mexicanos & 4576879 & 12484041 & 10565114 & 1918927 & 1002502 & 871816 & 4739489 & 3954183 \\
\hline Aguascalientes & 20733 & 90945 & 85344 & 5601 & 8803 & 8125 & 47217 & 41030 \\
\hline Baja California & 16291 & 88827 & 86198 & 2629 & 7752 & 7009 & 45718 & 38601 \\
\hline Baja California Sur & 15003 & 48782 & 46847 & 1935 & 4280 & 3532 & 21088 & 16371 \\
\hline Campeche & 41055 & 145079 & 108036 & 37043 & 12008 & 10697 & 65858 & 55328 \\
\hline Coahuila & 34264 & 182822 & 165537 & 17285 & 14387 & 12469 & 105064 & 87383 \\
\hline Colima & 17288 & 82841 & 76644 & 6197 & 4897 & 4167 & 35422 & 26925 \\
\hline Chiapas & 107933 & 376210 & 294003 & 82207 & 73424 & 67255 & 147286 & 132159 \\
\hline Chihuahua & 86934 & 232286 & 201264 & 31022 & 22300 & 19528 & 101058 & 84426 \\
\hline Distrito Federal & 116330 & 448085 & 426616 & 21469 & 31477 & 28506 & 223931 & 194141 \\
\hline Durango & 24817 & 132270 & 125186 & 7084 & 17098 & 15413 & 84468 & 73003 \\
\hline Guanajuato & 175720 & 656517 & 575592 & 80925 & 48827 & 43587 & 329765 & 281449 \\
\hline Guerrero & 375530 & 916795 & 630094 & 286701 & 78676 & 66500 & 229784 & 188685 \\
\hline Hidalgo & 126279 & 375274 & 303856 & 71418 & 21508 & 18712 & 189521 & 156852 \\
\hline Jalisco & 214794 & 627254 & 579684 & 47570 & 36933 & 33125 & 269603 & 228827 \\
\hline México & 1149698 & 2285935 & 2031017 & 254918 & 175623 & 148406 & 728886 & 591738 \\
\hline Michoacán & 108648 & 487821 & 430986 & 56835 & 44724 & 39601 & 219093 & 189504 \\
\hline Morelos & 51318 & 217041 & 184489 & 32552 & 20272 & 17673 & 112970 & 97035 \\
\hline $\mathrm{N}$ ayarit & 79991 & 175747 & 154301 & 21446 & 11603 & 9862 & 53893 & 42780 \\
\hline Nuevo León & 161699 & 410562 & 397891 & 12671 & 15590 & 13735 & 162268 & 139182 \\
\hline 0 axaca & 150064 & 477453 & 345143 & 132310 & 61970 & 55817 & 173536 & 148860 \\
\hline Puebla & 224310 & 557089 & 456561 & 100528 & 44943 & 39034 & 201751 & 163461 \\
\hline Q uerétaro & 74396 & 240045 & 194584 & 45461 & 15856 & 14284 & 100782 & 87481 \\
\hline Q uintana Roo & 41631 & 112134 & 80388 & 31746 & 7705 & 6682 & 46169 & 38946 \\
\hline San Luis Potosí & 158610 & 345005 & 251221 & 93784 & 17369 & 15364 & 93593 & 76499 \\
\hline Sinaloa & 82288 & 246256 & 230017 & 16239 & 20800 & 18266 & 90982 & 75552 \\
\hline Sonora & 52097 & 258040 & 250115 & 7925 & 24847 & 21447 & 123355 & 102830 \\
\hline Tabasco & 263700 & 605200 & 494557 & 110643 & 36297 & 29015 & 137821 & 105167 \\
\hline Tamaulipas & 81742 & 297361 & 277395 & 19966 & 22826 & 19732 & 122290 & 104491 \\
\hline Tlaxcala & 98498 & 241846 & 208060 & 33786 & 10373 & 8378 & 85264 & 66332 \\
\hline Veracruz & 261934 & 757270 & 612881 & 144389 & 47991 & 40828 & 208412 & 169999 \\
\hline Yucatán & 103253 & 266018 & 177661 & 88357 & 21674 & 18530 & 99000 & 81217 \\
\hline Zacatecas & 60031 & 99231 & 82946 & 16285 & 18465 & 15333 & 82939 & 67837 \\
\hline Institutos nacionales & 0 & 0 & 0 & 0 & 1204 & 1204 & 702 & 92 \\
\hline
\end{tabular}

Fuente: Sistema de Información en Salud para PoblaciónA bierta (SISPA), D irección General de Estadística e Informática (D G El), Subsecretaría de Prevención y Control de Enfermedades, Secretaría de Salud (SSA), México 


\section{Atencion materna. México, 1999}

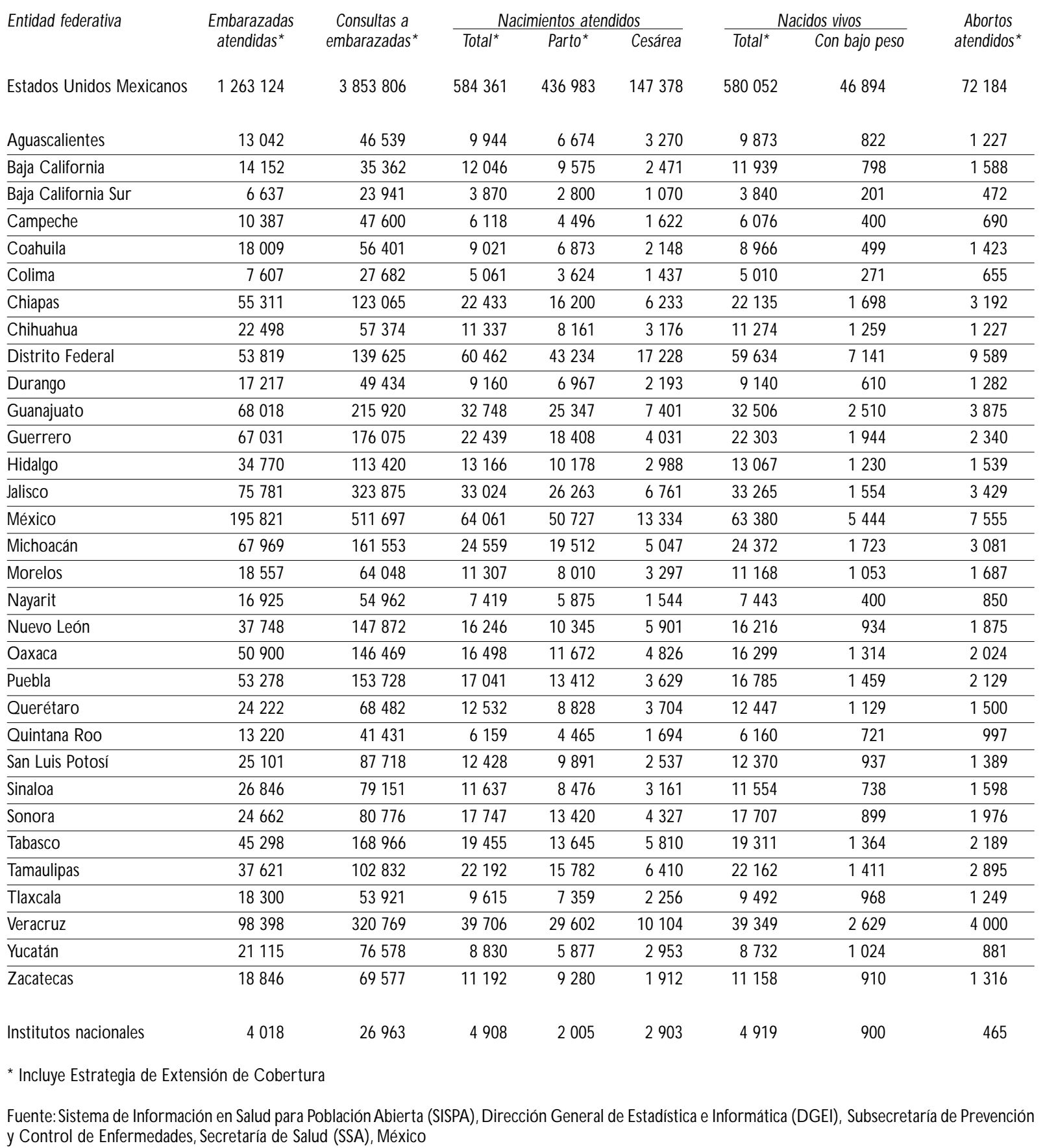




\section{Cuadro VI}

\section{Servicios de planificación familiar. MéXico, 1999}

\begin{tabular}{|c|c|c|c|c|c|c|c|}
\hline \multirow[t]{2}{*}{ Entidad federativa } & \multirow{2}{*}{$\begin{array}{l}\text { Consultas y } \\
\text { atenciones* }\end{array}$} & \multirow{2}{*}{$\begin{array}{l}\text { Nuevos } \\
\text { aceptantes* }\end{array}$} & \multirow{2}{*}{$\begin{array}{l}\text { Usuarias } \\
\text { activas* }\end{array}$} & \multicolumn{3}{|c|}{ Intervenciones quirúrgicas } & \multirow{2}{*}{$\begin{array}{l}\text { Aceptantes post } \\
\text { evento obstétrico }\end{array}$} \\
\hline & & & & Total & Oclusión tubaria & Vasectomía & \\
\hline Estados Unidos Mexicanos & 7998533 & 932852 & 2623024 & 102151 & 96509 & 5642 & 259341 \\
\hline Aguascalientes & 96106 & 8543 & 22671 & 1185 & 1077 & 108 & 2748 \\
\hline Baja C alifornia & 77220 & 16454 & 26369 & 1676 & 1518 & 158 & 4246 \\
\hline Baja California Sur & 41104 & 6121 & 16633 & 645 & 619 & 26 & 1624 \\
\hline Campeche & 82963 & 9615 & 23865 & 1959 & 1891 & 68 & 3715 \\
\hline Coahuila & 166491 & 18750 & 44880 & 1617 & 1522 & 95 & 5199 \\
\hline Colima & 54274 & 10066 & 23294 & 931 & 897 & 34 & 4042 \\
\hline Chiapas & 341625 & 40424 & 104130 & 5269 & 5175 & 94 & 15905 \\
\hline Chihuahua & 144548 & 17883 & 40213 & 1489 & 1349 & 140 & 4133 \\
\hline Distrito Federal & 251097 & 81295 & 114331 & 7836 & 6787 & 1049 & 14039 \\
\hline Durango & 154592 & 16215 & 65238 & 1529 & 1418 & 111 & 4795 \\
\hline Guanajuato & 314962 & 39861 & 94058 & 4081 & 3970 & 111 & 9728 \\
\hline Guerrero & 550450 & 40929 & 162899 & 5918 & 5737 & 181 & 8717 \\
\hline Hidalgo & 205603 & 25758 & 70391 & 2779 & 2592 & 187 & 5287 \\
\hline Jalisco & 416399 & 53329 & 149475 & 5052 & 4598 & 454 & 8597 \\
\hline México & 1695531 & 106120 & 517126 & 8434 & 7901 & 533 & 33604 \\
\hline Michoacán & 311914 & 45922 & 94289 & 3603 & 3426 & 177 & 9552 \\
\hline Morelos & 144928 & 17984 & 47801 & 2496 & 2387 & 109 & 5198 \\
\hline $\mathrm{N}$ ayarit & 136931 & 17614 & 38142 & 1273 & 1221 & 52 & 3273 \\
\hline Nuevo León & 207270 & 35072 & 78225 & 3050 & 2864 & 186 & 12613 \\
\hline 0 axaca & 266376 & 30023 & 86801 & 2990 & 2907 & 83 & 6973 \\
\hline Puebla & 522487 & 52415 & 146685 & 3725 & 3454 & 271 & 8903 \\
\hline Q uerétaro & 69172 & 12073 & 35873 & 1876 & 1772 & 104 & 4422 \\
\hline Q uintana Roo & 77953 & 9333 & 19302 & 1302 & 1199 & 103 & 1960 \\
\hline San Luis Potosí & 135344 & 20248 & 46784 & 1954 & 1851 & 103 & 6194 \\
\hline Sinaloa & 140181 & 25668 & 49581 & 2087 & 1959 & 128 & 4124 \\
\hline Sonora & 134601 & 18692 & 44509 & 2371 & 2137 & 234 & 4696 \\
\hline Tabasco & 245412 & 21252 & 113456 & 4635 & 4522 & 113 & 9847 \\
\hline Tamaulipas & 156825 & 21074 & 42283 & 3887 & 3700 & 187 & 17616 \\
\hline Tlaxcala & 109669 & 13054 & 47488 & 2213 & 2130 & 83 & 5437 \\
\hline Veracruz & 493169 & 72159 & 166131 & 9898 & 9746 & 152 & 22215 \\
\hline Yucatán & 98461 & 11255 & 38463 & 1825 & 1746 & 79 & 3944 \\
\hline Zacatecas & 149860 & 17651 & 51638 & 1396 & 1338 & 58 & 4144 \\
\hline Institutos nacionales & 5015 & 0 & 0 & 1170 & 1099 & 71 & 1851 \\
\hline
\end{tabular}




\section{A cCidentes atendidos. México, 1999}

\begin{tabular}{|c|c|c|c|c|c|c|c|}
\hline Entidad federativa & Total & $<5$ años & De 5 a 14 años & De 15 a 24 años & De 25 a 49 años & De 50 a 64 años & De 65 y más años \\
\hline Estados Unidos Mexicanos & 936832 & 114235 & 238688 & 207384 & 251185 & 75179 & 50161 \\
\hline Aguascalientes & 19844 & 2611 & 5267 & 4578 & 5014 & 1363 & 1011 \\
\hline Baja California & 13857 & 1381 & 2617 & 3364 & 4911 & 1014 & 570 \\
\hline Baja C alifornia Sur & 4934 & 565 & 1098 & 1236 & 1592 & 291 & 152 \\
\hline Campeche & 7680 & 688 & 1822 & 1961 & 2421 & 500 & 288 \\
\hline Coahuila & 15935 & 2129 & 4023 & 3360 & 4275 & 1330 & 818 \\
\hline Colima & 10399 & 949 & 2716 & 2585 & 2720 & 887 & 542 \\
\hline Chiapas & 37010 & 3432 & 7675 & 9289 & 11458 & 3367 & 1789 \\
\hline Chihuahua & 30064 & 3591 & 7075 & 6676 & 9139 & 2200 & 1383 \\
\hline Distrito Federal & 53124 & 6420 & 12824 & 12033 & 14645 & 4434 & 2768 \\
\hline Durango & 11313 & 1331 & 3187 & 2413 & 2779 & 941 & 662 \\
\hline Guanajuato & 60978 & 7550 & 15526 & 14475 & 14560 & 5219 & 3648 \\
\hline Guerrero & 60709 & 6552 & 16061 & 12560 & 16092 & 5691 & 3753 \\
\hline Hidalgo & 36711 & 3443 & 9959 & 7355 & 10045 & 3673 & 2236 \\
\hline Jalisco & 34569 & 4202 & 9495 & 7899 & 8411 & 2359 & 2203 \\
\hline México & 114974 & 15397 & 30787 & 25171 & 29815 & 8469 & 5335 \\
\hline Michoacán & 35029 & 4556 & 9181 & 7595 & 8922 & 2730 & 2045 \\
\hline Morelos & 42118 & 5806 & 11036 & 9608 & 10903 & 2743 & 2022 \\
\hline $\mathrm{N}$ ayarit & 14742 & 1680 & 3733 & 3276 & 3990 & 1161 & 902 \\
\hline Nuevo León & 18603 & 3549 & 6243 & 3037 & 3747 & 1138 & 889 \\
\hline 0 axaca & 46798 & 4133 & 12031 & 9727 & 13154 & 4614 & 3139 \\
\hline Puebla & 27962 & 2523 & 7503 & 6396 & 7454 & 2484 & 1602 \\
\hline Q uerétaro & 16031 & 1948 & 4226 & 3699 & 4200 & 1135 & 823 \\
\hline Q uintana Roo & 14917 & 1603 & 3414 & 3797 & 4717 & 883 & 503 \\
\hline San Luis Potosí & 16152 & 1633 & 4539 & 3243 & 4123 & 1447 & 1167 \\
\hline Sinaloa & 15686 & 2161 & 4153 & 3224 & 4115 & 1127 & 906 \\
\hline Sonora & 31178 & 4910 & 8204 & 6699 & 8335 & 1868 & 1162 \\
\hline Tabasco & 26884 & 2562 & 6557 & 6843 & 8184 & 1812 & 926 \\
\hline Tamaulipas & 23580 & 4638 & 5826 & 4740 & 5692 & 1667 & 1017 \\
\hline Tlaxcala & 12634 & 1328 & 2870 & 3282 & 3513 & 959 & 682 \\
\hline Veracruz & 49513 & 7495 & 11440 & 9865 & 13758 & 4358 & 2597 \\
\hline Yucatán & 18763 & 1892 & 4188 & 4401 & 5061 & 1822 & 1399 \\
\hline Zacatecas & 14141 & 1577 & 3412 & 2997 & 3440 & 1493 & 1222 \\
\hline
\end{tabular}

Fuente: Sistema de Información en Salud para PoblaciónA bierta (SISPA), D irección G eneral de Estadística e Informática (D G EI), Subsecretaría de Prevención y Control de Enfermedades, Secretaría de Salud (SSA), México 


\section{Detección de enfermedades. México, 1999}

\begin{tabular}{|c|c|c|c|c|c|c|c|c|}
\hline \multirow[t]{2}{*}{ Entidad federativa } & \multirow{2}{*}{$\begin{array}{c}\text { Cáncer } \\
\text { cervicouterino }\end{array}$} & \multirow{2}{*}{$\begin{array}{c}\text { Cáncer } \\
\text { de mama }\end{array}$} & \multicolumn{2}{|c|}{ Diabetes mellitus } & \multicolumn{2}{|c|}{ Hipertensión arterial } & \multicolumn{2}{|c|}{ Tuberculosis } \\
\hline & & & Hombres & Mujeres & Hombres & Mujeres & Hombres & M ujeres \\
\hline Estados Unidos Mexicanos & 2506205 & 3277711 & 1077026 & 2600443 & 2663836 & 6923407 & 167549 & 210685 \\
\hline Aguascalientes & 23821 & 25001 & 8787 & 21938 & 18327 & 53182 & 755 & 1004 \\
\hline Baja California & 38661 & 34517 & 15038 & 33069 & 36932 & 89384 & 3862 & 3344 \\
\hline Baja California Sur & 15897 & 17245 & 7400 & 18957 & 11974 & 28024 & 937 & 1135 \\
\hline Campeche & 33712 & 34037 & 10310 & 24517 & 16890 & 41165 & 591 & 791 \\
\hline Coahuila & 50556 & 57866 & 22611 & 56370 & 45440 & 118933 & 5175 & 7106 \\
\hline Colima & 21133 & 23585 & 11158 & 25699 & 13964 & 35396 & 4737 & 4849 \\
\hline Chiapas & 87059 & 136566 & 42384 & 84269 & 142106 & 321107 & 5228 & 6073 \\
\hline Chihuahua & 44149 & 53146 & 28234 & 57897 & 69687 & 144319 & 4137 & 3876 \\
\hline Distrito Federal & 178150 & 190433 & 72160 & 195488 & 210584 & 553046 & 3664 & 5007 \\
\hline Durango & 36883 & 53914 & 15787 & 40613 & 35267 & 104837 & 4361 & 4744 \\
\hline Guanajuato & 123211 & 147465 & 39102 & 117429 & 83853 & 262889 & 3779 & 5105 \\
\hline Guerrero & 155771 & 161244 & 80461 & 172293 & 152588 & 344400 & 9657 & 12508 \\
\hline Hidalgo & 96383 & 127042 & 46735 & 102936 & 132359 & 317152 & 7020 & 10658 \\
\hline Jalisco & 155362 & 158001 & 23434 & 61893 & 76045 & 217055 & 9888 & 11371 \\
\hline México & 159182 & 375851 & 99494 & 263878 & 332369 & 889830 & 5138 & 8463 \\
\hline Michoacán & 91570 & 118312 & 58054 & 140579 & 122920 & 317566 & 3698 & 5203 \\
\hline Morelos & 44545 & 49744 & 21825 & 44972 & 76935 & 170838 & 2397 & 3570 \\
\hline $\mathrm{N}$ ayarit & 41910 & 45132 & 16972 & 35747 & 44263 & 93554 & 6359 & 7197 \\
\hline N uevo León & 101879 & 174553 & 49989 & 147255 & 98231 & 322509 & 4879 & 6785 \\
\hline 0 axaca & 85012 & 132338 & 37276 & 70791 & 108515 & 241030 & 5487 & 7319 \\
\hline Puebla & 120905 & 195974 & 79941 & 178360 & 147282 & 363681 & 13114 & 18321 \\
\hline Q uerétaro & 42539 & 46306 & 11573 & 33472 & 41985 & 118737 & 1286 & 1628 \\
\hline Q uintana Roo & 26385 & 32914 & 8131 & 20436 & 26511 & 66168 & 1103 & 1018 \\
\hline San Luis Potosí & 73270 & 83553 & 38891 & 84038 & 49323 & 114451 & 3043 & 5242 \\
\hline Sinaloa & 77396 & 83107 & 18480 & 47364 & 52939 & 187235 & 7838 & 8918 \\
\hline Sonora & 60942 & 60833 & 27784 & 67638 & 58099 & 146522 & 7067 & 5480 \\
\hline Tabasco & 124031 & 132841 & 46230 & 107994 & 68097 & 181582 & 4658 & 5488 \\
\hline Tamaulipas & 71391 & 93134 & 28508 & 73279 & 88745 & 229869 & 8325 & 9289 \\
\hline Tlaxcala & 48195 & 64839 & 24060 & 62389 & 36098 & 97778 & 1201 & 2225 \\
\hline Veracruz & 180252 & 265698 & 48898 & 121450 & 201208 & 586546 & 23746 & 32710 \\
\hline Yucatán & 39484 & 34844 & 17435 & 37345 & 31485 & 74438 & 1816 & 2187 \\
\hline Zacatecas & 54839 & 67631 & 19884 & 50088 & 32815 & 90184 & 2131 & 2071 \\
\hline Institutos nacionales & 1730 & 45 & 0 & 0 & 0 & 0 & 472 & 0 \\
\hline
\end{tabular}

Fuente: Sistema de Información en Salud para Población A bierta (SISPA), D irección General de Estadística e Informática (D GEI), Subsecretaría de Prevención y Control de Enfermedades, Secretaría de Salud (SSA), México 